\title{
Postoperative Pulmonary Complications in Complex Paediatric and Adult Spine Deformity: A Retrospective Review of Consecutive Patients Treated at SRS GOP Site in Ghana
}

Irene Wulff

FOCOS Orthopaedic Hospital-Accra

Henry Ofori Duah ( $\square$ duahhenryofori@gmail.com )

FOCOS Orthopaedic Hospital https://orcid.org/0000-0002-4842-6006

Henry Osei Tutu

FOCOS Orthopaedic Hospital-Accra

Gerhard Ofori-Amankwah

FOCOS Orthopaedic Hospital-Accra

Kwadwo Poku Yankey

FOCOS Orthopaedic Hospital-Accra

Mabel Adobea Owiredu

FOCOS Orthopaedic Hospital-Accra

Halima Bidemi Yahaya

Zenith Medical and Kidney Center , Abuja

Harry Akoto

Korle Bu Teaching Hospital

Audrey Oteng-Yeboah

Korle Bu Teaching Hospital

FOCOS Spine Research Group

FOCOS Orthopaedic Hospital-Accra

Oheneba Boachie-Adjei

FOCOS Orthopaedic Hospital-Accra

\section{Research article}

Keywords: Complex spine deformity; Pulmonary complications; late presentation; Forced Vital Capacity; Halo Gravity Traction; FVC; pediatric deformity; scoliosis; Pulmonary function tests; PFT; complications; Preoperative management.

Posted Date: November 26th, 2019 
DOI: https://doi.org/10.21203/rs.2.17793/v1

License: (c) (1) This work is licensed under a Creative Commons Attribution 4.0 International License. Read Full License 


\section{Abstract}

Background Pulmonary complications are important cause of morbidity and mortality in patients following spinal surgeries. There is paucity of literature on pulmonary complications following complex spine deformity surgery in underserved regions. This study sought to assess the incidence and risk factors of pulmonary complication following complex spine deformity surgery Methods Data of 276 complex spine deformity patients aged 3-25yrs who were consecutively treated at a single site were retrospectively reviewed. Data was analyzed using Stata 14 software. Patients were labelled into two groups: Grp 1: patients with pulmonary complications $(N=17)$ vs $\mathrm{Grp} 2$ : patients with no pulmonary complications ( $\mathrm{N}=259)$. Comparative analysis for risk factors included independent $\mathrm{t}$-test and chi square test for independence. Multivariate logistic regression analysis was also performed. Results The incidence proportion of pulmonary complication was 17/276 (6.1\%) (Grp 1) whiles 259 pts had no pulmonary events (Grp 2). There were $8 \mathrm{M} / 9 \mathrm{~F}$ for Grp 1 vs $100 \mathrm{M} / 159 \mathrm{~F}$ Grp 2, p=0.48. BMI was similar in both groups (17.2 vs $18.4 \mathrm{~km}-2, p=0.15)$. Average pre-op sagittal cobb ( 90.6 vs $88.7 \mathrm{deg}, p=0.87$.), coronal cobb (95 vs 88.5deg, $p=0.43$ ), Pre-Op FVC (45.3 vs 62.0\%, $p=0.02$ ), Pre-Op FEV1 (41.9\% vs 63.1, $p<0.001$ ), Grp 1 vs Grp 2, respectively. EBL, OR time and Surgery Levels were similar in both Grps. Thoracoplasty was performed in $41.18 \%$ vs $21.57 \%$, $p=0.06$, SPO $47.06 \%$ vs $42.31 \%, p=0.04$ and VCR $5.88 \%$ vs $20.31 \%$, $\mathrm{p}=0.145$, Grp 1 vs Grp 2, respectively. Multivariate logistic regression showed that every unit increase in pre-Op FVC (\%) decreases the odds of pulmonary complication by $5 \%(\mathrm{OR}=0.95,95 \% \mathrm{Cl} 0.90$ to 0.99 , $\mathrm{p}=0.045$ ). Conclusion The observed $6.1 \%$ incidence of pulmonary complications is comparable to reported series. Only pre-Op FVC was an independent predictor of pulmonary complications. The observed case fatality rate (17\%) following pulmonary complications highlights the need for thorough preoperative evaluation to identify high risk patients. Key words: Complex spine deformity; Pulmonary complications; late presentation; Forced Vital Capacity; Halo Gravity Traction; FVC; pediatric deformity; scoliosis; Pulmonary function tests; PFT; complications; Preoperative management.

\section{Background}

The surgical management of complex spine deformity is technically challenging and associated with significant perioperative complications [1-8]. Post-operative(post-op) pulmonary complications remain one of the leading causes of mortality even in adult spine surgery [5-6]. The cumulative incidence of pulmonary complications after spine surgery has ranged from 0.9 to $9.8 \%$ [7-12]. The differences in incidence are attributable to the variations in the definition of pulmonary events in the various studies [712].

The concern for pulmonary complications is even greater in developing regions with limited expertise and resources. Patients in these regions typically report to health facilities when spine deformities have significantly progressed [13]. These severe deformities are associated with poor pulmonary function [1418]. Impaired pulmonary function increases the risk of post-op pulmonary complications after spine surgery [18]. Besides, spine surgery for these patients is characterized by significant blood loss, long operative time, and the use of three column osteotomies which further increases the risk for neurologic 
and non-neurologic complications [1]. The presence of comorbidities coupled with major surgical procedures further increases the risk of pulmonary complications [9]

The mechanism of pulmonary complication following spinal surgery may be related to direct trauma, embolization of marrow material into the lung including fat, ventilation-association lung injury and transfusion-associated lung injury [19-23]. Post-op pulmonary complications increase the risk of mortality after spine surgery $[5,6,7]$. Aside mortality, post-op pulmonary complications can affect the length of stay and cost of hospitalization [7]. Identification of risk factors for pulmonary complications would help avert these outcomes. However, there is paucity of literature on pulmonary complications following complex pediatric spine deformity surgery in underserved regions such as West Africa. This study sought to investigate the incidence and risk factors for pulmonary complications following complex pediatric and adult spine deformity surgeries in specialist Orthopaedic Hospital in Ghana, West Africa.

\section{Methods}

Data of 276 consecutive complex spine deformity patients aged 3-25yrs who were treated at a single site in Ghana from January, 2015 to December, 2017 were retrospectively reviewed using information from the FOCOS Spine Registry, electronic records and filed folders. Institutional Review Board (IRB) approval was obtained from the Noguchi Memorial Institute for Medical Research (NMIMR) prior to initiation of the study (Certified Protocol Number: 057/16-17). Research data was initially extracted in excel format and exported into Stata 14 Software for analysis. The primary outcome reviewed was post-op pulmonary complications which was defined as any pulmonary event following spine deformity surgery including respiratory distress, prolonged intubation of more than 48hrs, re-intubation, pneumonia, Acute respiratory distress syndrome (ARDS), significant pneumothorax and tracheostomy. Record review for the primary outcome was restricted to pulmonary complication events that occurred within the first six (6) weeks after surgery. All deformity etiology types were included in the analysis. Patients were divided into two groups during data analysis: Group 1: patients who developed pulmonary complications and Group 2: patients who did not develop pulmonary complications (Differences between the groups were not known prior to data collection). Comparative analysis was performed between the two groups to identify the risk factors. Multivariate logistic regression analysis was performed to evaluate the strength of the association.

\section{Results}

A total of 276 paediatric and adult deformity patients were reviewed. 94 patients (34.06\%) were aged 310yrs, 141 patients (51.09\%) were aged 11-17yrs, and 41 patients (14.86\%) were aged 18-25yrs. Surgical procedures included posterior only in 262 patients, combined anterior and posterior in 7 patients and 7 two-staged posterior only performed at average 1 week apart. Etiology of the deformities was Idiopathic in 145 patients (52.54\%), Congenital in 59 patients (21.38\%), Post-tubercular (Post TB) in 44 patients (15.94\%), Neuromuscular in 14 patients (5.07\%), and Neurofibromatosis in 14 patients (5.07\%). For curve type, 154 patients (55.8\%) had Scoliosis, 60 patients (21.74\%) had Kyphosis and $62(22.46 \%)$ had 
kyphoscoliosis. A total of 17 patients had post-op pulmonary complication (Group 1) representing a cumulative incidence of $6.16 \% .259$ patients had no pulmonary complications (Group 2). Gender ratio $(\mathrm{M} / \mathrm{F})$ was similar in both groups: 0.89 versus $(\mathrm{vrs})$ 0.63. Mean age were 13.2 years $( \pm 5.0)$ and 12.5 years $( \pm 4.48)$ in Group 1 and Group 2, respectively $(p=0.55)$.

The mean pre-operative (pre-op) Body Mass Index (BMI) was comparable in Group 1 ( $M=17.2$ $\left.\mathrm{kg} / \mathrm{m}^{2} \pm 2.31\right)$ and Group $2\left(M=18.4 \mathrm{~kg} / \mathrm{m}^{2} \pm 3.49, \mathrm{p}=0.15\right)$. Pre-op sagittal cobb angles were similar in both groups $\left(M=90.6^{\circ} \pm 39\right.$ vrs $\left.M=88.7^{\circ} \pm 48, p=0.87\right)$, respectively. The range of pre-op sagittal cobb angles were $41-203^{\circ}$ (Group1) vrs $11-263^{\circ}$ Group 2. Average pre-op coronal cobb in Group 1 was $95^{\circ}$ $( \pm 24.68)$ compared to $88.5^{\circ}( \pm 32.28)$ in Group $2(p=0.43)$. The range of pre-op coronal cobb angles were $56-144^{\circ}$ (Group1) vrs $18-231^{\circ}$ (Group 2).

In terms of pulmonary function, pre-op Forced vital capacity (FVC) (\%) was significantly lower in Group 1 $(M=45.3 \pm 15.59)$ compared to Group $2(M=62.0 \pm 24.69, p=0.02)$. Likewise, pre-op forced expiratory volume in one second (FEV1) (\%) was lower in Group $1(\mathrm{M}=41.9 \pm 15.45)$ compared to group $2(\mathrm{M}=63.1 \pm$ 21.13, $p<0.001$ ). Post traction FVC and FEV1 were similar in both groups. (Table 1).

The average duration of surgery $(\mathrm{min})$ in Group $1(\mathrm{M}=183.7 \pm 63.09)$ was comparable to that of Group 2 $(M=189.6 \pm 80.72, p=0.77)$. Average number of levels fused in Group $1(M=13.87 \pm 2.63)$ was similar to that of Group $2(M=12.46 \pm 2.99, p=0.07)$. The average estimated blood loss ( $E B L)$ in Group $1(M=1021 \mathrm{mls}$ \pm 683 ) was comparable to Group $2(\mathrm{M}=1011 \mathrm{mls} \pm 911, \mathrm{p}=0.93)$. In terms of osteotomies, Smith-Petersen osteotomy (SPO) was performed in $47 \%$ of patients in Group 1 compared to $42 \%$ of patients in Group 2 $(p=0.04)$, and vertebral column resection (VCR) was performed in $5.88 \%$ of patients in Group 1 compared to $20.31 \%$ in Group 2 ( $p=0.145$ ). Thoracoplasty was performed in $41.18 \%$ and $21.57 \%$ in Group 1 and Group 2, respectively $(p=0.06)$. (Table 1$)$

Table 1: Summary of demographic, clinical and surgical logs by post-op pulmonary complication status 


\begin{tabular}{|c|c|c|c|}
\hline \multirow[t]{3}{*}{ Category } & \multicolumn{2}{|c|}{$\begin{array}{l}\text { Post-operative Pulmonary Complication } \\
\text { Status }\end{array}$} & \multirow[t]{3}{*}{ p-value } \\
\hline & Yes (Group 1) & No (Group 2 ) $\mathbf{N}=(259)$ & \\
\hline & $\mathbf{N}=17$ & & \\
\hline \multicolumn{4}{|l|}{ Age } \\
\hline 3-10yrs & $4(23.53 \%)$ & $90(34.75 \%)$ & \\
\hline 11-17yrs & $10(58.82 \%)$ & $131(50.58 \%)$ & \\
\hline $18-25 y r s$ & $3(17.75 \%)$ & $38(14.67 \%)$ & 0.69 \\
\hline Gender (Male/Female) & $8 / 9(0.89)$ & $100 / 159(0.63)$ & 0.48 \\
\hline $\mathrm{BMI}\left(\mathrm{kg} / \mathrm{m}^{2}\right)$ & $17.2 \pm 2.31$ & $18.4 \pm 3.49$ & 0.15 \\
\hline \multicolumn{4}{|l|}{ Etiologies } \\
\hline Idiopathic & $7(41.18 \%)$ & $138(53.28 \%)$ & \\
\hline Congenital & $5(29.41 \%)$ & $54(20.85 \%)$ & \\
\hline Post Tuberculous & $1(5.88 \%)$ & $43(16.60 \%)$ & \\
\hline Neuromuscular & $2(11.76 \%)$ & $12(4.63)$ & \\
\hline \multicolumn{4}{|l|}{ Neurofibromatosis } \\
\hline Pre-op sagittal cobb & $90.6 \pm 39$ & $88.7 \pm 48$ & 0.87 \\
\hline Pre-op coronal cobb & $95 \pm 24.68$ & $88.5 \pm 32.28$ & 0.43 \\
\hline Post traction sagittal cobb & $78.0 \pm 22.6$ & $84.3 \pm 38.6$ & 0.60 \\
\hline \multicolumn{4}{|l|}{$\begin{array}{l}\text { Post traction coronal } \\
\text { cobb }\end{array}$} \\
\hline Pre-Op FVC (\%) & $45.3 \pm 15.59$ & $62.0 \pm 24.69$ & $0.02 * * *$ \\
\hline Pre-Op FEV1 (\%) & $41.9 \pm 15.45$ & $63.1 \pm 21.13$ & $<0.001^{* * *}$ \\
\hline Post-Traction FVC & $46 \pm 14.93$ & $55.45 \pm 18.65$ & 0.10 \\
\hline Post-Traction FEV1 & $45.73 \pm 15.68$ & $54.84 \pm 16.27$ & 0.08 \\
\hline $\mathrm{EBL}(\mathrm{ml})$ & $1021 \pm 683$ & $1011 \pm 911$ & 0.93 \\
\hline Duration of Surgery (min) & $183.7 \pm 63.09$ & $189.6 \pm 80.72$ & 0.77 \\
\hline Surgery Levels & $13.87 \pm 2.63$ & $12.46 \pm 2.99$ & 0.07 \\
\hline \multirow{2}{*}{$\begin{array}{l}\text { Thoracoplasty } \\
\text { SPO }\end{array}$} & 7/17(41.18\%) & 55/255 (21.57\%) & 0.06 \\
\hline & $8 / 17(47.06 \%)$ & $62 / 255(42.31 \%)$ & $0.04^{* * *}$ \\
\hline VCR & $1 / 17(5.88 \%)$ & $52 / 256(20.31 \%)$ & 0.145 \\
\hline \multicolumn{4}{|c|}{ *** means significant at 0.05 alpha level. } \\
\hline \\
\hline \multicolumn{4}{|c|}{ Categorical variables are reported as frequencies (column \%) } \\
\hline \multicolumn{4}{|c|}{ BMI: Body Mass Index } \\
\hline \multicolumn{4}{|l|}{ FVC: Forced vital capacity } \\
\hline \multicolumn{4}{|c|}{ FEV1: Forced Expiratory Volume in one second (FEV1) } \\
\hline \multicolumn{4}{|l|}{ EBL: Estimated Blood Loss } \\
\hline \multicolumn{4}{|l|}{ SPO: Smith-Peterson Osteotomy } \\
\hline VCR: Vertebral Column Resection & & & \\
\hline
\end{tabular}

\section{Predictors of Pulmonary Complications in Multivariate Analysis}

Only Pre-operative FVC was found to be a significant independent predictor of pulmonary complication in multivariate analysis. Multivariate logistic regression revealed that every $1 \%$ increase in pre-operative FVC 
decreases the odds of pulmonary complication by $5 \%(\mathrm{OR}=0.95,95 \% \mathrm{Cl} 0.90$ to $0.99, \mathrm{p}=0.045)$, after controlling for age, curve type, curve etiology, BMI, gender, pre-op sagittal and coronal cobb angles, duration of surgery, EBL, thoracoplasty, SPO, PSO and VCR. (Table 2)

\section{Table 2: Multivariate Logistic Regression Estimates of the Risk Factors for Pulmonary Complications}

\begin{tabular}{|c|c|c|c|c|}
\hline Table 2 & & & & \\
\hline Risk factors & Odds Ratio & 95\% Confi & e Interval & p-value \\
\hline & & Lower & Upper & \\
\hline AGE & 1.11 & 0.86 & 1.43 & 0.427 \\
\hline $\mathrm{BMI}$ & 0.85 & 0.65 & 1.11 & 0.227 \\
\hline Gender (Female) & 0.53 & 0.11 & 2.43 & 0.413 \\
\hline & & & & \\
\hline Curve Type & & & & \\
\hline Scoliosis & Ref. & - & - & - \\
\hline Kvphosis & 1 & & & \\
\hline Kyphoscoliosis & 1.20 & 0.13 & 11.00 & 0.870 \\
\hline Etiology & & & & \\
\hline Idiopathic & Ref. & - & - & - \\
\hline Congenital & 2.94 & 0.33 & 25.80 & 0.331 \\
\hline Post Tuberculous & 1 & & & \\
\hline Neuromuscular & 2.81 & 0.14 & 54.56 & 0.494 \\
\hline Neurofibromatosis & 2.12 & 0.13 & 33.12 & 0.591 \\
\hline Pre Op Coronal Cobb & 0.98 & 0.94 & 1.02 & 0.460 \\
\hline Pre Op Sagittal Cobb & 1.01 & 0.98 & 1.04 & 0.613 \\
\hline Pre-Op FVC & 0.95 & 0.90 & 0.99 & $0.045^{\star \star \star}$ \\
\hline EBL & 0.99 & 0.99 & 1.00 & 0.823 \\
\hline Duration of surgery & 0.98 & 0.96 & 1.00 & 0.084 \\
\hline Thoracoplasty & 5.19 & 0.40 & 67.94 & 0.209 \\
\hline $\mathrm{SPO}$ & 0.83 & 0.10 & 6.70 & 0.868 \\
\hline PSO & 1 & & & \\
\hline VCR & 1 & & & \\
\hline $\begin{array}{l}\text { *** means significant at } 0 . \\
\text { BMI: Body Mass Index } \\
\text { FVC: Forced vital capacity } \\
\text { EBL: Estimated Blood Loss } \\
\text { SPO: Smith-Peterson Oste } \\
\text { PSO: pedicle subtraction } o \\
\text { VCR: Vertebral Column Re }\end{array}$ & $\begin{array}{l}\text { omy } \\
\text { teotomy } \\
\text { ection }\end{array}$ & & & \\
\hline
\end{tabular}

\section{Outcomes of Pulmonary Events}


The etiologies of the 17 patients who developed pulmonary complications comprised 7 Idiopathic, 5 congenital, 2 Neuromuscular, 2 Neurofibromatosis and 1 Post TB. There were 3 mortalities (1 congenital, 1 Post TB and 1 Neurofibromatosis) representing a case fatality rate of $17.6 \%$. The details of the outcomes of pulmonary events by type of pulmonary event and deformity etiologies are shown in Table 3 and Table 4, respectively.

\section{Table 3: Outcomes of Pulmonary Complications by type of Pulmonary Event}

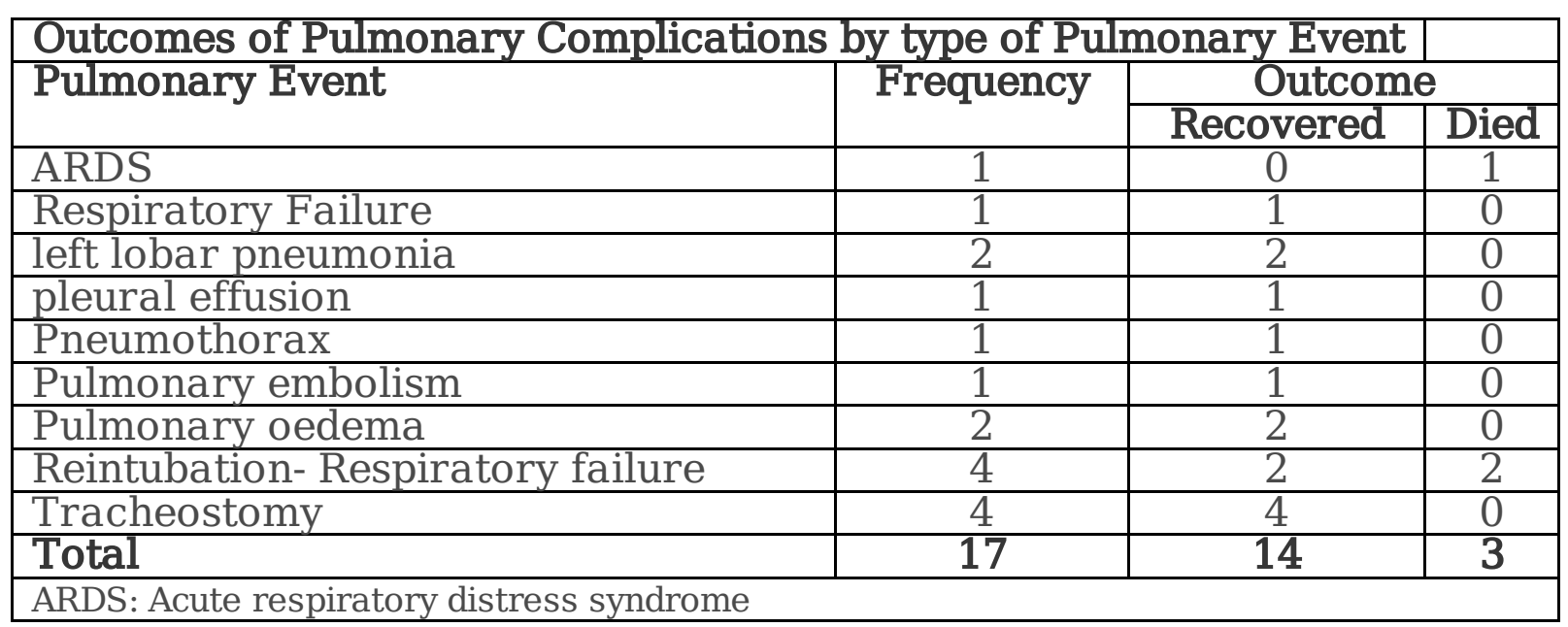

\section{Table 4: Outcomes of Pulmonary Complications by Curve Etiologies}

\begin{tabular}{|l|c|c|c|}
\hline Table 4 & \multicolumn{2}{|c|}{ Outcome } \\
\hline Pulmonary Event & \multirow{2}{*}{ Frequency } & \multicolumn{2}{|c|}{} \\
\cline { 3 - 4 } & & Recovered & Died \\
\hline Idiopathic & 7 & 7 & 0 \\
\hline Congenital & 5 & 4 & 1 \\
\hline Post Tuberculous & 1 & 0 & 1 \\
\hline Neuromuscular & 2 & 2 & 0 \\
\hline Neurofibromatosis & 2 & 1 & 1 \\
\hline Total & 17 & 14 & 3 \\
\hline \multicolumn{3}{|l|}{} \\
\hline
\end{tabular}

\section{Discussion}

Pulmonary complications are very critical in perioperative management of patients after complex deformity surgery. Despite the variation in definition of pulmonary complications, the conditions classified as such are generally similar and they include Empyema, Haemothorax, Pleural effusion, Postop hypoxia, Pneumonia, Pneumothorax, Pulmonary embolus, Respiratory arrest, Transfusion-related Acute Lung Injury (TRALI), Transfusion Associated Circulatory Overload (TACO) and pulmonary edema amongst other pulmonary problems $[9,19,20,24-26]$.

The cumulative incidence of post-op pulmonary complication observed in this cohort was $6.16 \%$. This incidence is within the range reported by previous studies [7-12]. Patil et al. [7] reported a post-op 
pulmonary complication rate of $8.1 \%$ among idiopathic patients < $18 y$ ears (1-17yrs) old and $9.8 \%$ in idiopathic patients $>18 y$ years old (18-84yrs). Imposti et al. [9] also reported a $9 \%$ cumulative incidence of a pulmonary complication after spine surgery. Their patients were $>18$ years and comprised degenerative and trauma cases. Moreover, pulmonary complications within 2-year period after spinal surgery were included in their reported cumulative incidence [9]. However, the present study included only paediatric and young adult deformity patients aged 3-25years and reported only early pulmonary complications (up to 6 weeks post-op). These methodological variations could account for the slight difference observed in the cumulative incidence of post-op pulmonary complications.

It is already established that complex spine deformity is associated with poor pulmonary function [14-17]. However, the degree of pulmonary compromise is not attributable to curve magnitudes alone [17]. We observed that pre-op FVC (\% predicted) was significantly lower in patients who developed pulmonary complications compared to those who had no pulmonary complications. Further analysis with multivariate logistic regression revealed that increasing pre-op FVC was associated with reduced odds of pulmonary complications ( $\mathrm{OR}=0.95,95 \% \mathrm{Cl} 0.90$ to $0.99, \mathrm{p}=0.035)$. Spine surgery can cause significant transient decline in PFT up to $60 \%$ of pre-op values with a gradual return to baseline 1-2 months after surgery [27]. Therefore, patients with poor baseline PFT may have higher risk for post-op pulmonary complications because they are likely to experience further deterioration in their PFT after spine surgery compared to their counterparts with good baseline PFT. Lao et al. [18] also reported that patients with severe or moderate preoperative pulmonary dysfunction, as measured by the FVC ratio, had a higher incidence of post-op pulmonary complications. Stein et al. [24] also found a higher incidence of pulmonary complications in patients with abnormal PFT results compared to those with normal PFTs. In addition, Kang et al. [25] found that neuromuscular patients with FVC $<39.5 \%$ have increased risk of postop pulmonary complications following spine surgery. Preoperative long term Halo gravity traction with breathing exercises may help in this regard.

We report a case-fatality rate of $17.6 \%$ among patients who developed post-op pulmonary complications. This highlights the likelihood of an adverse outcome when there is a pulmonary complication. Previous studies have also documented significant mortality following post-op pulmonary complications $[5,6,9,26]$. Imposti et al. [9] found that mortality rate from pulmonary complications was 3.61 per 1,000 persons per year. Pumberger et al. [26] also assessed the risk factors for in-hospital mortality following lumbar fusion surgery and found that patients who developed post-op pneumonia had 3 times greater risk of mortality whiles those who developed pulmonary embolism had 10 times greater risk of mortality. The case-fatality rate observed in the present series of paediatric and young adult spine deformity patients underscores the need for proactive perioperative measures to reduce pulmonary complications.

The $6.16 \%$ incidence of pulmonary complication recorded in this series is commendable for a private facility in low-resource setting such as Ghana. Various perioperative measures have enabled us to achieve such comparable pulmonary complication rates despite the complexities of the spine deformity cases present in this cohort. These included utilization of pre-op Halo Gravity Traction (HGT) [13,28-29], 
adequate pre-op nutritional rehabilitation [30], elective overnight ventilation after surgery for patients with poor FVC and early application of tracheostomy at the earliest sign of difficulty in weaning off ventilation.

Therefore, we make the following recommendations for surgeons performing complex deformity surgery in low-resource settings:

1. Identification of high risk patients with poor pre-op FVC

2. Utilization of HGT to reduce curve magnitude and stiffness

3. Pre-op nutritional optimization

4. Elective post-op ventilation and use of prophylactic diuretics for pulmonary edema

5. Application of Tracheotomy at the earliest sign of respiratory distress

6. Need for ICU coverage

\section{Strengths and Limitations}

This paper is the largest single site review of post-operative pulmonary complications following complex spine deformity surgery in Africa. However, as a retrospective study, the study has certain limitations. Analysis and findings were based on only data that could be retrieved. The study reported on early pulmonary complications (up 6weeks post-op) hence it is possible that the complication rate may vary if patients were followed over longer period after surgery. These patients are being followed for long term pulmonary outcomes.

\section{Conclusion}

We report a $6.16 \%$ cumulative incidence of pulmonary complication in our series which is within the range of other reports. Pre-op FVC was the only significant independent risk factor for pulmonary complications. The high case fatality rate observed (17.6\%) implies the need for thorough pre-op evaluation to identify high risk patients and the need for proactive perioperative measures to reduce incidence and mortality associated with pulmonary complications.

\section{List Of Abbreviations}

BMl: Body Mass Index

FVC: Forced vital capacity

FEV1: Forced Expiratory Volume in one second (FEV1)

Post-op: Post-operative

Pre-op: Pre-operative

EBL: Estimated Blood Loss 
SPO: Smith-Peterson Osteotomy

VCR: Vertebral Column Resection

Post TB: Post-tubercular

TRALI: Transfusion-related Acute Lung Injury

TACO: Transfusion Associated Circulatory Overload ()

ARDS: Acute respiratory distress syndrome

HGT: Halo Gravity Traction

IRB: Institutional Review Board

NMIMR: Noguchi Memorial Institute for Medical Research

OR: Odds Ratio

\section{Declarations}

\section{Ethics approval and consent to participate}

Institutional Review Board (IRB) approval was obtained from the Noguchi Memorial Institute for Medical Research (NMIMR) to use de-identified secondary data from the FOCOS Spine Registry (Certified Protocol Number: 057/16-17 amend. 2018). No additional consents were obtained from patients.

\section{Consent for publication}

Not Applicable.

\section{Availability of data and materials}

The datasets used and/or analyzed during the current study are available from the corresponding author on reasonable request.

\section{Competing interests}

OBA (grants, personal fees and other from K2M, other from WEIGAO, outside the submitted work). For the remaining authors none were declared.

\section{Funding}

This study received no specific grant from any funding agency in public, commercial or not-for-profit sectors. 


\section{Authors' contributions}

IW, HOD, OBA, GOA, KPY, AOY, HA and the FSRG designed the study. IW, HOD and OBA contributed to the writing of the introduction. HOD, HOT, MAO, HBY, AOY and FSRG contributed to data verification and cleaning. HOD performed the data analysis. IW, HOD, HBY, MAO, KPY, GOA, HA, AOY and HOT contributed to interpretation of statistical results. HOD, IW and OBA contributed to writing the methods section. HOD, IW, and OBA contributed to the writing of the discussion. All authors reviewed the final manuscript. All authors read and approved the final manuscript.

\section{Acknowledgements}

The authors acknowledge other members of the FOCOS Spine Research Group (FSRG) who contributed in diverse ways at various stages of the paper but are not listed, by names, as authors to this study.

\section{Authors' information.}

IW is the Chief of Anaesthesia and the Associate Medical Director at the FOCOS Orthopaedic Hospital, Ghana.

HOD is a graduate nurse and holds a Master of Public Health (MPH) degree from the Department of Epidemiology, School of Public Health, University of Ghana. He is currently the Clinical Research Coordinator at the FOCOS Orthopaedic Hospital, Ghana.

HOT has a BSc in Health Informatics and serves as a Clinical Research Officer at FOCOS Orthopaedic Hospital, Ghana.

MAO has a master's degree in Health Administration and serves as a Research Officer at FOCOS Orthopaedic Hospital, Ghana.

GOA is a Family Physician and serves as the director of medical services at the FOCOS Orthopaedic Hospital, Ghana.

KPY is a consultant neurosurgeon at the FOCOS Orthopaedic Hospital, Ghana.

HBY is a consultant Anaesthetist at the Zenith Medical and Kidney Center, Abuja-Nigeria

HA is a consultant neurosurgeon at the Korle-Bu Teaching Hospital, Accra-Ghana

AOY consultant Anaesthetist at the Korle-Bu Teaching Hospital, Accra-Ghana

Oheneba Boachie-Adjei is a Professor emeritus of Orthopaedic Surgery and Founder \& President of FOCOS Orthopaedic Hospital.

FSRG is collaborative and multidisciplinary group of researchers who conduct research into the management of complex paediartric and adult spine deformities at the FOCOS Orthopaedic Hospital. 


\section{References}

1. Boachie-Adjei O, Yagi M, Nemani VM, Sacramento-Dominguez C, Akoto H, Cunningham ME, et al. Incidence and risk factors for major surgical complications in patients with complex spinal deformity: A report from an SRS GOP site. Spine Deform. 2015;3(1):57-64.

2. Boachie-Adjei O, Yagi M, Sacramento-Dominguez C, Akoto H, Cunningham ME, Gupta M, Hess WF, Lonner BS, Ayamga J, Papadopoulus E, Sanchez-Perez-Grueso F. Surgical risk stratification based on preoperative risk factors in severe pediatric spinal deformity surgery. Spine deformity. 2014 Sep 1;2(5):340-9.

3. Nasser R, Yadla S, Maltenfort MG, Harrop JS, Anderson DG, Vaccaro AR, Sharan AD, Ratliff JK. Complications in spine surgery: a review. Journal of Neurosurgery: Spine. 2010 Aug;13(2):144-57.

4. Coe JD, Arlet V, Donaldson W, Berven S, Hanson DS, Mudiyam R, Perra JH, Shaffrey Cl. Complications in spinal fusion for adolescent idiopathic scoliosis in the new millennium. A report of the Scoliosis Research Society Morbidity and Mortality Committee. Spine. 2006 Feb 1;31(3):345-9.

5. Pumberger M, Chiu YL, Ma Y, Girardi FP, Vougioukas V, Memtsoudis SG. Perioperative mortality after lumbar spinal fusion surgery: an analysis of epidemiology and risk factors. European Spine Journal. 2012 Aug 1;21(8):1633-9.

6. Memtsoudis SG, Vougioukas VI, Ma Y, Gaber-Baylis LK, Girardi FP. Perioperative morbidity and mortality after anterior, posterior and anterior/posterior spine fusion surgery. Spine. 2011 Oct 15;36(22):1867

7. Patil CG, Santarelli J, Lad SP, Ho C, Tian W, Boakye M. Inpatient complications, mortality, and discharge disposition after surgical correction of idiopathic scoliosis: a national perspective. The Spine Journal. 2008 Nov 1;8(6):904-10.

8. Deyo R A, Cherkin D C, Loeser J D. et al . Morbidity and mortality in association with operations on the lumbar spine. The influence of age, diagnosis, and procedure. J Bone Joint Surg Am. 1992; 74(4) 536-543

9. Imposti F, Cizik A, Bransford R, Bellabarba C, Lee MJ. Risk factors for pulmonary complications after spine surgery. Evidence-based spine-care journal. 2010 Aug;1(2):26.

10. Carreon LY, Puno RM, Dimar JR, Glassman SD, Johnson JR. Perioperative complications of posterior lumbar decompression and arthrodesis in older adults. JBJS. 2003 Nov 1;85(11):2089-92.

11. Kalanithi P S, Patil C G, Boakye M. National complication rates and disposition after posterior lumbar fusion for acquired spondylolisthesis. Spine. 2009; 34(18) 1963-1969

12. Vaidya R, Carp J, Bartol S, Ouellette N, Lee S, Sethi A. Lumbar spine fusion in obese and morbidly obese patients. Spine. 2009 Mar 1;34(5):495-500.

13. Nemani VM, Kim HJ, Bjerke-Kroll BT, Yagi M, Sacramento-Dominguez C, Akoto H, Papadopoulos EC, Sanchez-Perez-Grueso F, Pellise F, Nguyen JT, Wulff I. Preoperative halo-gravity traction for severe spinal deformities at an SRS-GOP site in West Africa: protocols, complications, and results. Spine. 2015 Feb 1; 40(3):153-61. 
14. Johari J, Sharifudin MA, Ab Rahman A, Omar AS, Abdullah AT, Nor S, Lam WC, Yusof MI. Relationship between pulmonary function and degree of spinal deformity, location of apical vertebrae and age among adolescent idiopathic scoliosis patients. Singapore medical journal. 2016 Jan;57(1):33.

15. Lin MC, Liaw MY, Chen WJ, Cheng PT, Wong AM, Chiou WK. Pulmonary function and spinal characteristics: their relationships in persons with idiopathic and postpoliomyelitic scoliosis. Archives of physical medicine and rehabilitation. 2001 Mar 31;82(3):335-41.

16. Wekre LL, Kjensli A, Aasand K, Falch JA, Eriksen EF. Spinal deformities and lung function in adults with osteogenesis imperfecta. The clinical respiratory journal. 2014 Oct 1;8(4):437-43

17. Menon B, Aggarwal B. Influence of spinal deformity on pulmonary function, arterial blood gas values, and exercise capacity in thoracic kyphoscoliosis. Neurosciences. 2007 Oct 1;12(4):293-8.

18. Lao L, Weng X, Qiu G, Shen J. The role of preoperative pulmonary function tests in the surgical treatment of extremely severe scoliosis. Journal of orthopaedic surgery and research. 2013 Dec;8(1):32.

19. Stundner O, Taher F, Pawar A, Memtsoudis SG. Pulmonary complications after spine surgery. World journal of orthopedics. 2012 Oct 18;3(10):156.

20. Benson AB. Pulmonary complications of transfused blood components. Critical Care Nursing Clinics. 2012 Sep 1;24(3):403-18.

21. Toy P, Gajic O, Bacchetti P, Looney MR, Gropper MA, Hubmayr R, Lowell CA, Norris PJ, Murphy EL, Weiskopf RB, Wilson G. Transfusion-related acute lung injury: incidence and risk factors. Blood. 2012 Feb 16;119(7):1757-67.

22. Stroud MH, McCarthy RE, Parham DM, Schexnayder SM. Fatal pulmonary fat embolism following spinal fusion surgery. Pediatric Critical Care Medicine. 2006 May 1;7(3):263-6.

23. Urban MK, Jules-Elysee KM, Beckman JB, Sivjee K, King T, Kelsey W, Boachie-Adjei O. Pulmonary injury in patients undergoing complex spine surgery. The Spine Journal. 2005 May 1;5(3):269-76.

24. Stein, M., Koota, G. M., Simon, M., \& Frank, H. A. (1962). Pulmonary evaluation of surgical patients. Jama, 181(9), 765-770.

25. Kang GR, Suh SW, Lee IO. Preoperative predictors of postoperative pulmonary complications in neuromuscular scoliosis. Journal of Orthopaedic Science. 2011 Mar 1; 16(2):139-47.

26. Pumberger M, Chiu YL, Ma Y, Girardi FP, Vougioukas V, Memtsoudis SG. Perioperative mortality after lumbar spinal fusion surgery: an analysis of epidemiology and risk factors. European Spine Journal. 2012 Aug 1;21(8):1633-9.

27. Yuan N, Fraire JA, Margetis MM, Skaggs DL, Tolo VT, Keens TG. The effect of scoliosis surgery on lung function in the immediate postoperative period. Spine. 2005 Oct 1;30(19):2182-5.

28. Iyer S, Boachie-Adjei O, Duah HO, Yankey KP, Mahmud R, Wulff I, Tutu HO, Akoto H. Halo Gravity Traction Can Mitigate Preoperative Risk Factors and Early Surgical Complications in Complex Spine Deformity. Spine. 2019 May 1;44(9):629-36. 
29. Iyer S, Duah HO, Wulff I, Tutu HO, Mahmud R, Yankey KP, Akoto H, Boachie-Adjei O. The Use of Halo Gravity Traction in the Treatment of Severe Early Onset Spinal Deformity. Spine. 2019 Jul 15;44(14):E841-5.

30. Opoku H, Yirerong T, Osei-Onwona B, Boachie-Adjei O, FOCOS Spine Research Group. The Use of Arm Span as a Substitute for Height in Calculating Body Mass Index (BMI) for Spine Deformity Patients. Spine deformity. 2018 Jun 30;6(3):220-5. 